\title{
An early improvement threshold to predict response and remission in first-episode schizophrenia
}

R. Schennach-Wolff, F. H. Seemüller, A. Mayr, W. Maier, S. Klingberg, I. Heuser, J. Klosterkötter, M. Gastpar, H. Häfner, H. Sauer, F. Schneider, W. Gaebel, M. Jäger, H.-J. Möller and M. Riedel

\section{Background}

Early improvement with treatment is thought to be important in patients with first-episode schizophrenia, yet a valid definition is still outstanding.

\section{Aims}

To develop a valid definition of early improvement and test its predictive validity regarding response and remission.

\section{Method}

We examined 188 in-patients with first-episode schizophrenia. Early improvement was defined as improvement in Positive and Negative Syndrome Scale (PANSS) total score at week 2, response as a 40\% PANSS total score improvement at end-point, and remission according to consensus criteria.

\section{Results}

Reasonable predictive validity of early improvement was found for a 46\% PANSS total score improvement at week 2 and a $50 \%$ improvement for remission (area under the curve: response 0.707 , remission 0.692). Estimated confidence intervals ranged from 26 to $62 \%$ PANSS reduction for response and remission.

\section{Conclusions}

Patients with a first episode of schizophrenia should improve by at least 30\% in PANSS total score at week 2 to achieve response and remission.

\section{Declaration of interest}

W.G. has received/is receiving research grants/support from, serves as a consultant or is on the advisory board for, or is a member of the speaker bureau for Astrazeneca, BristolMyers Squibb, GlaxoSmithKline, Janssen-Cilag, Lilly Deutschland, Lundbeck, Novartis Pharma, Sanofi-Synthelabo/ Aventis and Wyeth Pharma. M.G. is a consultant or member of the advisory board for AstraZeneca, Lundbeck, Pfizer, Servier and Wyeth. M.J. has received honoraria and travel payments from AstraZeneca, Janssen-Cilag and Eli Lilly. H.J.M. has received/is receiving research grants/support from, serves as a consultant or is on the advisory board for or is a member of the speaker bureau for Astrazeneca, Bristol-Myers Squibb, Eli Lilly, Eisai, GlaxoSmithKline, JanssenCilag, Lundbeck, Merck, Novartis, Organon, Pfizer, Sanofi Aventis, Sepracor, Servier and Wyeth. M.R. has received research grants/support or has served as a consultant for AstraZeneca, Pfizer, Otsuka Pharma and Janssen-Cilag. In the context of investigator-initiated trials, M.R. has received support from AstraZeneca and Pfizer. F.S. has received/is receiving research grants/support from, serves as a consultant or is on the advisory board for Astrazeneca, Janssen-Cilag, Lundbeck Neuroscience and Wyeth.
According to the early-onset hypothesis of antipsychotic drug action, ${ }^{1}$ absence of an early response to medication is a stable predictor of subsequent lack of response in patients with schizophrenia. ${ }^{2-4}$ Despite the importance of identifying early improvement with treatment in first-episode schizophrenia, ${ }^{5}$ to our knowledge only a limited number of trials have investigated this topic. Emsley et al found response at week 6 to be one of the most stable predictors of remission after 2 years and 4 years. $^{6,7}$ Crespo-Facorro et al analysed acute treatment response in 172 patients and concluded that the identification of non-responders is the first step towards optimising therapeutic efforts. ${ }^{8}$ However, to date no systematic analysis finding the best definition of early response and concurrently evaluating its predictive validity for subsequent outcome has been carried out. In addition, although first-generation and second-generation antipsychotics have demonstrated a similar likelihood of achieving a favourable response in patients with first-episode schizophrenia, ${ }^{9}$ data on early response have yet to be reported. Therefore, to expand prior research, we developed a valid definition of early treatment improvement that best predicts treatment response and remission. Additionally, early improvement was compared between risperidone and haloperidol treatment.

\section{Method}

\section{Sample}

The patient sample was drawn from a multicentre follow-up programme performed within the German Research Network on Schizophrenia. ${ }^{10,11}$ The entire study programme consisted of an 8 -week acute study and a subsequent 2-year long-term treatment phase. The programme was conducted at 13 German university psychiatric hospitals according to the principles of good clinical practice and the Declaration of Helsinki. The study reported here covers the 8-week acute treatment phase.

Participants were selected from all individuals admitted to the in-patient departments between November 2000 and May 2004. Inclusion criteria were acute manifestation of a first episode of schizophrenia according to ICD-10 F20 criteria; age 18-65 years; adequate proficiency in the German language; no involuntary in-patient treatment up to the date of inclusion; and provision of written informed consent. ${ }^{10}$ Exclusion criteria were pregnancy; unchanged psychopathological condition or worsening of symptoms during pre-treatment with risperidone and haloperidol; other contraindications for risperidone or haloperidol; intellectual disability; organic brain disease; substance misuse; history of 
suicidal behaviour; severe physical disease; or participation in other trials. Approval was obtained from the coordinating centre as well as the local centres.

\section{Treatment}

Patients who had already been treated with psychotropic drugs underwent a 4-7 day wash-out period. After random assignment to treatment groups in a $1: 1$ ratio, patients received $2 \mathrm{mg}$ of the study medication once daily. Investigators, staff and patients were masked to treatment assignment. The dose could be increased by $1-2 \mathrm{mg}$ per day between day 3 and day 7 if required, and then at each weekly assessment up to a maximum of $8 \mathrm{mg}$ per day, but the total dose was not to exceed $4 \mathrm{mg}$ per day by week 2 . Psychotropic substances were not allowed as concomitant medication, with the exception of short-acting benzodiazepines for insomnia and lorazepam to arrest agitation and psychotic anxiety. The anticholinergic drug biperiden (up to $6 \mathrm{mg}$ per day) could be prescribed for extrapyramidal symptoms and the beta-blocker propranolol (up to $80 \mathrm{mg}$ per day) could be prescribed for akathisia.

\section{Assessments}

The diagnoses were established by clinical researchers on the basis of the German version of the Structured Clinical Interview for DSM-IV. ${ }^{12}$ During interviews with patients, relatives and care providers, a standardised documentation system was used to collect sociodemographic and course-related variables such as age at onset or duration of untreated psychosis. ${ }^{13}$ To assess symptom severity, the Positive and Negative Syndrome Scale (PANSS) for schizophrenia was used. ${ }^{14}$ Adverse events were assessed using the Abnormal Involuntary Movement Scale (AIMS). ${ }^{15}$ The Global Assessment of Functioning (GAF) scale and the Social and Occupational Functioning Assessment Scale (SOFAS) were applied to measure the patients' psychological, social and occupational functioning. ${ }^{12,16}$ Ratings were performed within 3 days of admission to hospital, and weekly during in-patient treatment until treatment week 8 . Interrater reliability yielded a satisfactory to good concordance which fitted to values of other publications, for example the intraclass correlation coefficient of the PANSS positive scale was $0.74(P<0.001)$.

\section{Statistical analysis}

Early improvement was defined as a percentage of any improvement in PANSS total score from baseline to week 2. Response was defined in accordance with Kinon et al as a minimum $40 \%$ reduction in PANSS total score from admission to week $8 .{ }^{17}$ For all calculations of percentage change the minimum of the PANSS total score was subtracted. ${ }^{18}$ Remission was defined according to the criteria recently proposed by the Remission in Schizophrenia Working Group as a PANSS rating of 3 or less on the following items: delusions (P1), unusual thought contents (G9), hallucinatory behaviour (P3), conceptual disorganisation (P2), mannerism/ posturing (G5), blunted affect (N1), social withdrawal (N4) and lack of spontaneity (N6). ${ }^{19}$ The time criterion of the applied consensus remission criteria could not be considered owing to the designated study duration of 8 weeks. Group differences regarding the outcome criteria were tested using Fisher's exact test for count data; the Wilcoxon rank sum test was applied for continuous data.

The discriminative and predictive ability of early improvement was evaluated using a receiver operating characteristic (ROC) curve. This curve combines the information of the true positive rate and the true negative rate. The area under the curve (AUC) is a measure of the overall discriminative power. A value of 0.5 for the AUC does not represent discriminative ability, whereas a value of 1.0 indicates perfect power. ${ }^{20}$ The optimal cut-off point is detected by maximising the sum of the true positive and true negative rates. To analyse AUC group differences a permutation test was applied. Permutation tests can be used in order to test the difference in the AUC values of a single metric variable in independent sample groups; the main advantage of this procedure is that no assumption about the distribution of the test statistics is needed.

In order to identify the most useful criterion of early improvement, total accuracy, sensitivity, specificity, and positive and negative predictive values were calculated for all possible early improvement cut-offs of a percentage PANSS total score improvement $(1 \%, 2 \% \ldots 98 \%, 99 \%)$. The total accuracy shows the proportion of patients whose psychopathological status at week 2 (early improvement or no early improvement) predicted subsequent response and remission at week $8 .^{21}$ Sensitivity is defined as the correct identification of patients subsequently achieving response or remission, and specificity as the correct identification of patients subsequently not achieving response or remission. The positive predictive value is the probability that a patient with early improvement will show subsequent response and remission, and the negative predictive value reflects the probability that a patient without early improvement will show subsequent non-response and non-remission.

When estimating a confidence interval for the optimum cutoff, the optimal cut-off point is detected by maximising the sum of sensitivity and specificity. To compute a confidence interval around this parameter, an assumption regarding the distribution of the latter is needed. To avoid this, the distribution can be simulated based on the data from the sample. This is commonly known as the classical non-parametric bootstrap procedure. All statistical analyses were performed using the statistical program $\mathrm{R}$ version 2.6.1 for Windows. ${ }^{22}$

\section{Results}

The patient sample available comprised 289 patients (for details see Möller et al). ${ }^{11}$ For the analysis reported here, 101 patients had to be excluded: 56 patients $(19 \%)$ discontinued the study within the first 3 weeks of treatment and for 45 patients (16\%) single items were missing in the requested PANSS data. Patients excluded and patients continuing the study were compared regarding sociodemographic, psychopathological and further clinical variables (Table 1). In total, 188 patients - 111 men (59\%) and 77 women (41\%) - were examined in this analysis; 93 patients were treated with risperidone and 95 with haloperidol. The applied mean dosages of risperidone and haloperidol are shown in Table 2. The mean age was 31.16 years (s.d.=9.09).

\section{Treatment response and remission}

According to the applied response criteria, 119 patients (63\%) were classified as treatment responders at week 8 and 116 patients $(62 \%)$ fulfilled remission criteria. Half the patients $(n=94)$ achieved response as well as remission, 25 patients responded but were not concurrently in remission and 22 patients achieved remission without concurrently being responders.

Divided into the two treatment groups, 59 haloperidoltreated patients (62\%) achieved response and 56 (59\%) achieved remission, with 46 patients being responders and remitters concurrently. In the risperidone group, 60 patients (64\%) fulfilled response criteria and 60 patients (64\%) fulfilled remission criteria, with 48 patients achieving response and remission concurrently. When the two patient subgroups were compared regarding differences in response and remission rates, no significant 


\begin{tabular}{|c|c|c|c|}
\hline & Patients excluded $(n=56)$ & Patients included $(n=188)$ & $P$ (adjusted) \\
\hline Age, years: mean (s.d.) & $27.18(8.98)$ & $31.16(10.00)$ & $0.0034(0.0548)$ \\
\hline \multicolumn{4}{|l|}{ PANSS score: mean (s.d.) } \\
\hline Positive scale & $21.98(7.89)$ & $21.17(5.89)$ & $0.5384(0.1000)$ \\
\hline Negative scale & $21.53(9.04)$ & $18.53(8.05)$ & $0.0352(0.2469)$ \\
\hline Global & $41.75(15.06)$ & $37.44(12.29)$ & $0.0593(0.2469)$ \\
\hline Total & $85.25(28.76)$ & $77.14(23.00)$ & $0.0617(0.2469)$ \\
\hline Insight into illness (G12): mean (s.d.) & 3.35 (1.78) & $3.24(1.64)$ & $0.7833(0.1000)$ \\
\hline GAF score: mean (s.d.) & $47.30(13.11)$ & $44.27(13.81)$ & $0.1007(0.3221)$ \\
\hline SOFAS score: mean (s.d.) & $47.20(12.68)$ & $45.69(13.17)$ & $0.3599(0.8226)$ \\
\hline DUP, months: mean (s.d.) & $3.08(2.26)$ & $2.94(2.13)$ & $0.7556(0.1000)$ \\
\hline Female, $n$ & 23 & 77 & $0.1000(0.1000)$ \\
\hline Medication with haloperidol, $n$ & 29 & 95 & $0.8803(1.0000)$ \\
\hline Pre-treatment, $n$ & 18 & 56 & $0.7426(1.0000)$ \\
\hline AlMS score $>0$ in week $1, n$ & 1 & 15 & $0.1288(0.2577)$ \\
\hline
\end{tabular}

\begin{tabular}{|c|c|c|c|c|}
\hline & \multicolumn{3}{|c|}{ Dosage, mg: mean (s.d.) } & \multirow[b]{2}{*}{$P^{\mathrm{a}}$} \\
\hline & Total & Haloperidol & Risperidone & \\
\hline Week 1 & $2.6(1.0)$ & $2.6(1.0)$ & $2.7(1.0)$ & 0.524 \\
\hline Week 2 & $3.6(1.3)$ & $3.5(1.3)$ & $3.7(1.3)$ & 0.203 \\
\hline Week 3 & $4.2(1.6)$ & $4.1(1.5)$ & $4.3(1.6)$ & 0.365 \\
\hline Week 4 & $4.5(1.8)$ & $4.5(1.8)$ & $4.5(1.9)$ & 0.847 \\
\hline Week 5 & $4.6(2.0)$ & $4.6(2.0)$ & $4.6(2.0)$ & 0.934 \\
\hline Week 6 & $4.7(2.1)$ & $4.6(2.1)$ & $4.8(2.2)$ & 0.523 \\
\hline Week 7 & $4.5(2.2)$ & $4.3(2.0)$ & $4.7(2.4)$ & 0.256 \\
\hline Week 8 & $4.4(2.2)$ & $4.0(2.0)$ & $4.6(2.4)$ & 0.151 \\
\hline
\end{tabular}

difference could be observed (for response $P=0.76$, for remission $P=0.46$ ). Additionally, when patients with an increase of dosage in the first two treatment weeks $(n=138)$ were compared regarding the achievement of response and remission, no significant association was found (for response $P=0.22$, for remission $P=1.00$ ).

\section{Improvement in PANSS total score at week 2}

The allocation of improvement depends on the definition applied, i.e. the required percentage reduction in PANSS total score after 2 weeks of treatment. For total sample and treatment subgroups see Table 3. No significant difference in the improvement in PANSS total score at week 2 could be detected between the risperidone and haloperidol treatment subgroups.

\section{Validity of improvement at week 2 as predictor of response} and remission

Response. To examine the ability of the psychopathological improvement at week 2 to predict response at week 8 , a ROC curve was generated for the total patient sample (AUC $=0.707$ ) and both treatment subgroups (haloperidol $\mathrm{AUC}=0.677$, risperidone $\mathrm{AUC}=0.737$ ) for the reduction in PANSS total score after the first two treatment weeks (Fig. 1). When comparing the validity of early improvement as a predictor of response between the two treatment groups, no significant difference could be observed $(P=0.43)$.
Remission. To examine the ability of improvement at week 2 to predict remission at week 8 , a ROC curve was generated for the total patient sample $(\mathrm{AUC}=0.692)$ and patient subgroups (haloperidol $\mathrm{AUC}=0.744$, risperidone $\mathrm{AUC}=0.638$ ) for the reduction in PANSS total score after the first two treatment weeks (Fig. 2). When the validity of early improvement as a predictor of remission was compared between the two treatment groups, no significant difference could be observed $(P=0.16)$.

\section{Optimum cut-off points of early improvement as predictor}

Response. Total accuracy, sensitivity, specificity, and positive and negative predictive values of different cut-off points (percentage improvement in PANSS total score) for predicting response at week 8 are shown in online Table DS1. A cut-off point of $46 \%$ showed the highest score for sensitivity (55\%) and specificity ( $80 \%$ ); the positive predictive value was $82 \%$ and the negative predictive value $50 \%$.

Remission. Total accuracy, sensitivity, specificity, and positive and negative predictive values of different cut-off points for predicting remission at week 8 are shown in online Table DS2. A cut-off point of $50 \%$ showed the highest score for sensitivity $(47 \%)$ and specificity $(85 \%)$; the positive predictive value was $83 \%$ and the negative predictive value $50 \%$.

Optimum cut-off estimated by a confidence interval using bootstrap Response. The bootstrap procedure led to a cut-off confidence interval of $26-60 \%(0.258-0.606)$. 


\begin{tabular}{|c|c|c|c|c|c|}
\hline $\begin{array}{l}\text { PANSS total score } \\
\text { improvement }\end{array}$ & $\begin{array}{c}\text { Total sample } \\
n(\%)\end{array}$ & $\begin{array}{c}\text { Haloperidol group } \\
n(\%)\end{array}$ & $\begin{array}{l}\text { Risperidone group } \\
\qquad n(\%)\end{array}$ & Odds ratio & $P$ \\
\hline $10 \%$ & 155 (82) & 77 (81) & $78(84)$ & 0.82 & 0.7025 \\
\hline $20 \%$ & $136(72)$ & $68(72)$ & $68(73)$ & 0.93 & 0.8711 \\
\hline $30 \%$ & $114(61)$ & $57(60)$ & 57 (61) & 0.95 & 0.8822 \\
\hline $40 \%$ & 89 (47) & 47 (49) & $42(45)$ & 1.19 & 0.5629 \\
\hline $50 \%$ & $66(35)$ & $33(35)$ & $33(36)$ & 0.97 & 1.0000 \\
\hline $60 \%$ & $53(28)$ & $27(29)$ & $26(27)$ & 1.09 & 0.8716 \\
\hline $70 \%$ & 31 (16) & $17(18)$ & $14(15)$ & 1.12 & 0.5593 \\
\hline $80 \%$ & $12(6)$ & $6(6)$ & $6(6)$ & 1.02 & 1.0000 \\
\hline $90 \%$ & $4(2)$ & $1(1)$ & $3(3)$ & 0.34 & 0.6210 \\
\hline $100 \%$ & $2(1)$ & $0(0)$ & $2(2)$ & 0.00 & 0.4974 \\
\hline
\end{tabular}

Remission. The bootstrap procedure led to a cut-off confidence interval of $28-62 \%(0.276-0.615)$.

As the $95 \%$ confidence intervals for the optimal cut-offs in both treatment groups heavily overlap and include the optimal cut-offs, one can assume that those cut-off points and therefore their intervals do not significantly differ, with $P>0.05$ between risperidone and haloperidol. ${ }^{23}$

Comparison of patients achieving or not achieving the proposed $30 \%$ improvement at week 2

The confidence intervals of a $26-60 \%$ PANSS total score improvement to predict response and a $28-61 \%$ improvement to predict remission seem rather impractical. Therefore, we propose a $30-60 \%$ reduction as definition of early improvement, suggesting that a patient should improve by at least $30 \%$ in PANSS total score. The time course of the PANSS total score comparing patients with early improvement and those without early improvement (30\% reduction) is shown in Fig. 3. Patients achieving this cut-off $v$. those not achieving it were further compared regarding the mean dosage of antipsychotic medication prescribed (Fig. 4). Patients with early improvement were significantly more likely to become responders $(P<0.001)$ as well as remitters $(P<0.01)$ compared with patients not achieving the

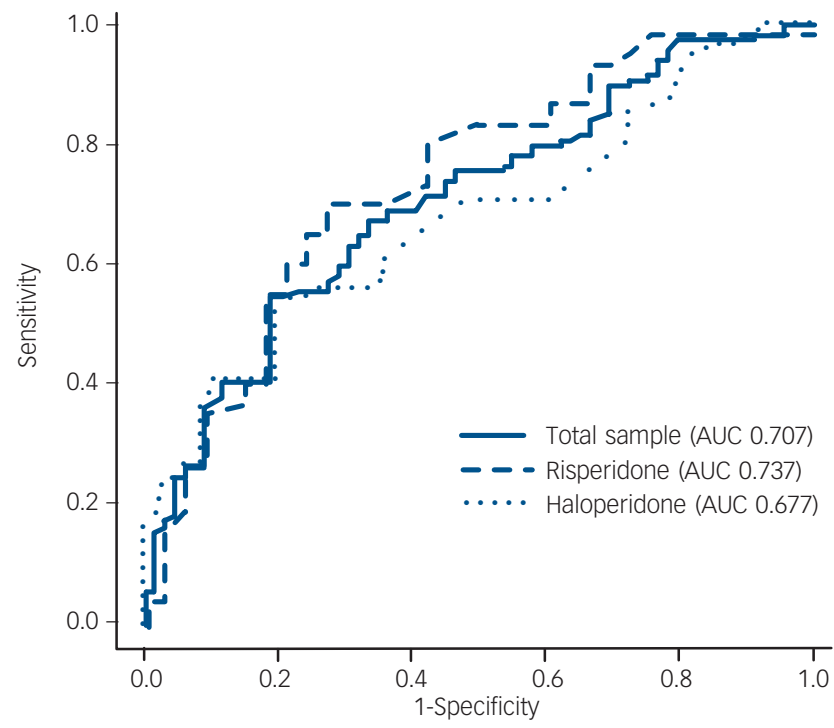

Fig. 1 Receiver operating characteristic curve for early improvement as predictor of response at discharge. AUC, area under the curve.
$30 \%$ improvement. No significant difference was found in a comparison of the two subgroups in terms of duration of current hospital treatment $(P=0.72)$ and gender $(P=0.29)$. Patients with early improvement were found to be significantly older than patients not achieving early improvement $(P<0.01)$.

Patients fulfilling the proposed improvement criteria at week 2 and achieving subsequent response and remission

Patient subgroups were formed according to achievement of the proposed cut-offs (numerical cut-off, lower and upper cut-off of the proposed cut-off interval) and subsequent response (Fig. 5) and remission (Fig. 6).

\section{Discussion}

The rates of treatment response (63\% of patients) and remission (62\% of patients) at week 8 are in good agreement with those reported by other authors. ${ }^{24}$ Additionally, we were able to replicate results of recent studies demonstrating that early improvement is a valid predictor of subsequent response and remission. ${ }^{2,3,25,26}$ It is well known that patients with a first psychotic episode will more often respond to antipsychotic treatment and show a favourable treatment outcome compared with those who have multiple

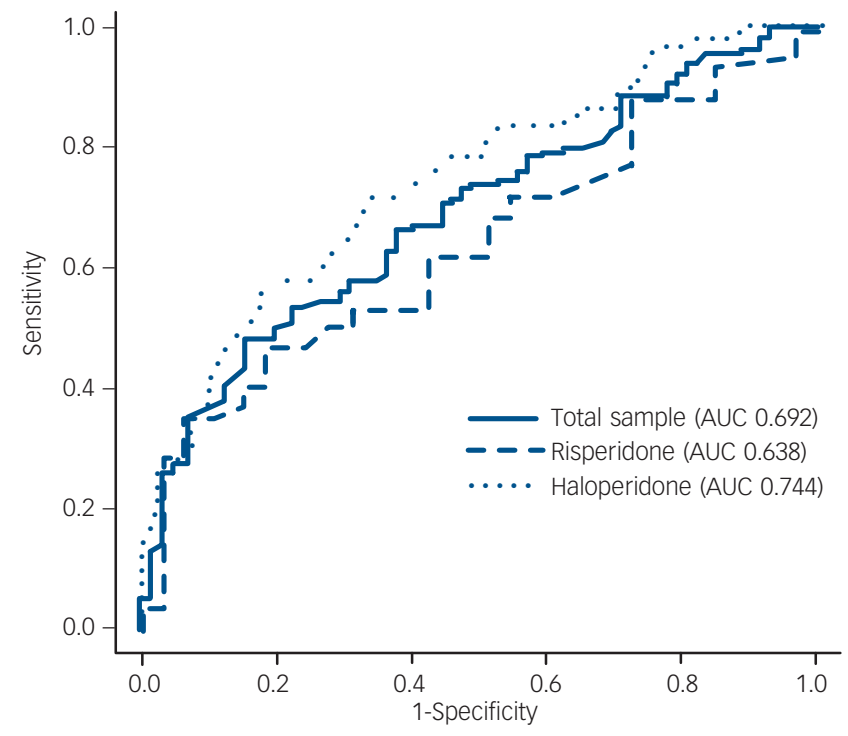

Fig. 2 Receiver operation characteristic curve for early improvement as predictor of remission at discharge. AUC, area under the curve. 


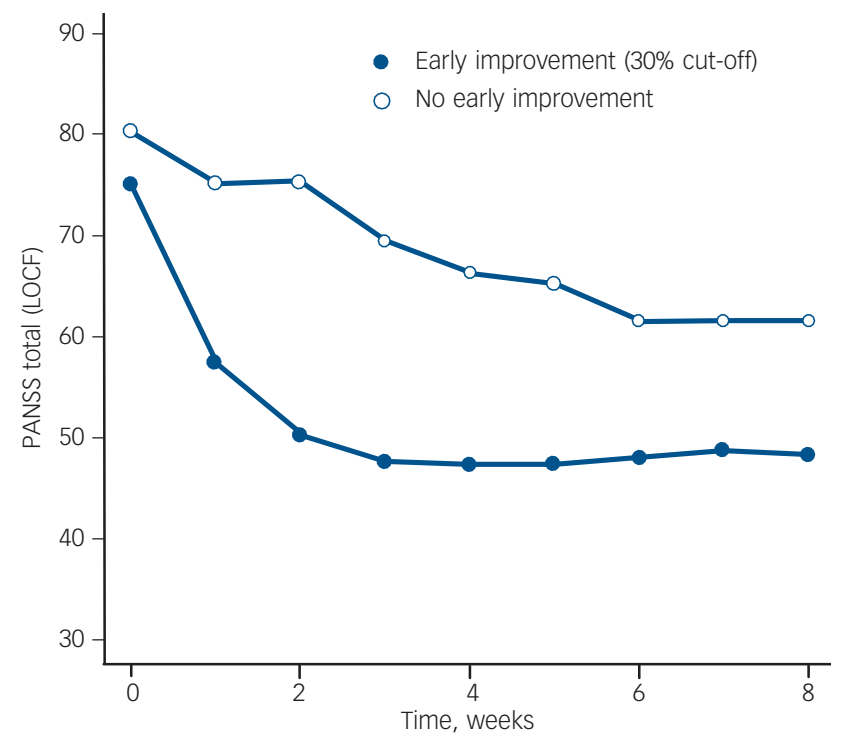

Fig. 3 Comparison of mean Positive and Negative Syndrome Scale (PANSS) total scores of patients achieving or not achieving the proposed $30 \%$ cut-off score. LOCF, last observation carried forward.

episodes. ${ }^{27}$ Almost $80 \%$ of the examined patients achieving response or remission achieved both outcome criteria concurrently, which might be surprising at first. Again, this may be due to the homogeneity of the patient sample, and to the fact that only the symptom severity component of the remission criteria was applied. Comparative literature examining response and concurrent remission is sparse. However, results from the European First Episode Schizophrenia Trial report response and remission rates differing by about $15-20 \%{ }^{28}$ It has to be kept in mind, however, that patients had to maintain the symptom severity component for 12 months rather than 8 weeks as in our study, which might explain the different results.

\section{Comparison of first- and second-generation antipsychotics}

We found no significant difference between first- and secondgeneration antipsychotics in response or remission rates, or concerning the predictive validity of early improvement. When examining the percentage reduction in the PANSS total score at week 2 we found no significant difference between the two medication groups, suggesting no difference with respect to early improver rates. These results resemble data deriving from clinical as well as double-blind controlled trials which have found almost no significant differences in efficacy when comparing a first- and a second-generation antipsychotic. ${ }^{9,29,30}$

\section{Developing a valid definition of early improvement as predictor}

Different definitions of early response, such as a Brief Psychiatric Rating Scale positive score reduction of 4 at week 1 to best predict response at week $4,{ }^{4}$ or a $<20 \%$ or $>20 \%$ PANSS total score improvement at week 2 to best predict subsequent response, ${ }^{2,25}$ point out current inconsistencies in the definition of such response. In addition, some proposed cut-off points were chosen arbitrarily, emphasising the need for a systematic analysis. Therefore, sensitivity and specificity analyses were performed to find the most suitable cut-off that predicts response and remission. At week 2, a $46 \%$ improvement in the PANSS total

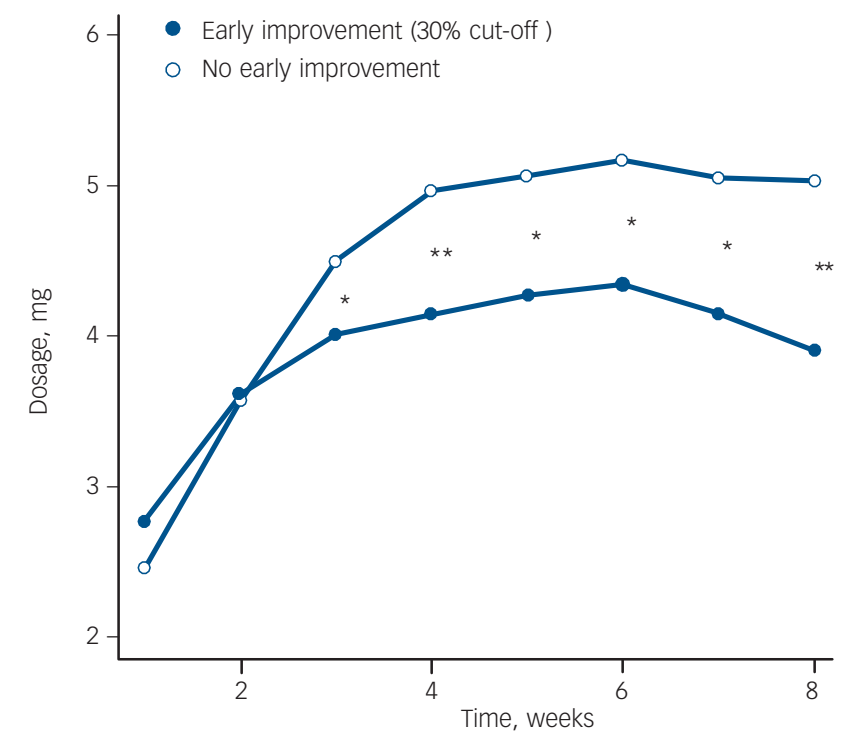

Fig. 4 Comparison of mean antipsychotic dosages applied in patients achieving or not achieving the proposed $30 \%$ cut-off score. ${ }^{*} P<0.05,{ }^{*} P<0.01$ (Wilcoxon rank sum test).

score was found to best predict response and a $50 \%$ improvement to best predict remission.

\section{Proposal of a cut-off interval as definition of early improvement}

Despite the common use of such sensitivity and specificity ROC analyses, this procedure also has its disadvantages. The main problem is that the results often cannot be generalised because they depend heavily on the patient sample. Even small changes in the data can change the resulting cut-off enormously. In our view this can also explain the diversity of proposed definitions of early improvement. In order to adjust for the dependency of the underlying sample we additionally computed bootstrap intervals by using resample methods. ${ }^{31}$ The results are confidence intervals that by definition cover the true cut-off for the population of patients with a first psychotic episode with a probability of 95\%. In addition, a cut-off interval provides a minimum score, meaning that this percentage reduction in the PANSS total score should at least be achieved by the patient. By presenting an upper range the cut-off interval demonstrates that there is a variety of appropriate cut-offs to predict subsequent outcome and with this it indicates a trend more than an all-or-nothing condition.

\section{Interval of $30-60 \%$ of early improvement}

Statistical analysis found a confidence interval of a 26-60\% PANSS total score improvement to predict response and a confidence interval of a $28-61 \%$ improvement to predict remission. Owing to the heavily overlapping intervals it appears that response and remission can be predicted using the same range. This can partly be explained by finding similar response and remission rates. Besides, in first-episode schizophrenia early improvement seems almost equally important regarding treatment response and improvement in core symptoms.

As mentioned above, since both $26 \%$ and $28 \%$ are impractical, we suggest instead an interval of $30-60 \%$. Our results indicate that a patient with a first psychotic episode should achieve at least a $30 \%$ PANSS total score improvement after 2 weeks to best predict treatment response or remission. This empirically derived cut-off value is high compared with recently proposed cut-off values 

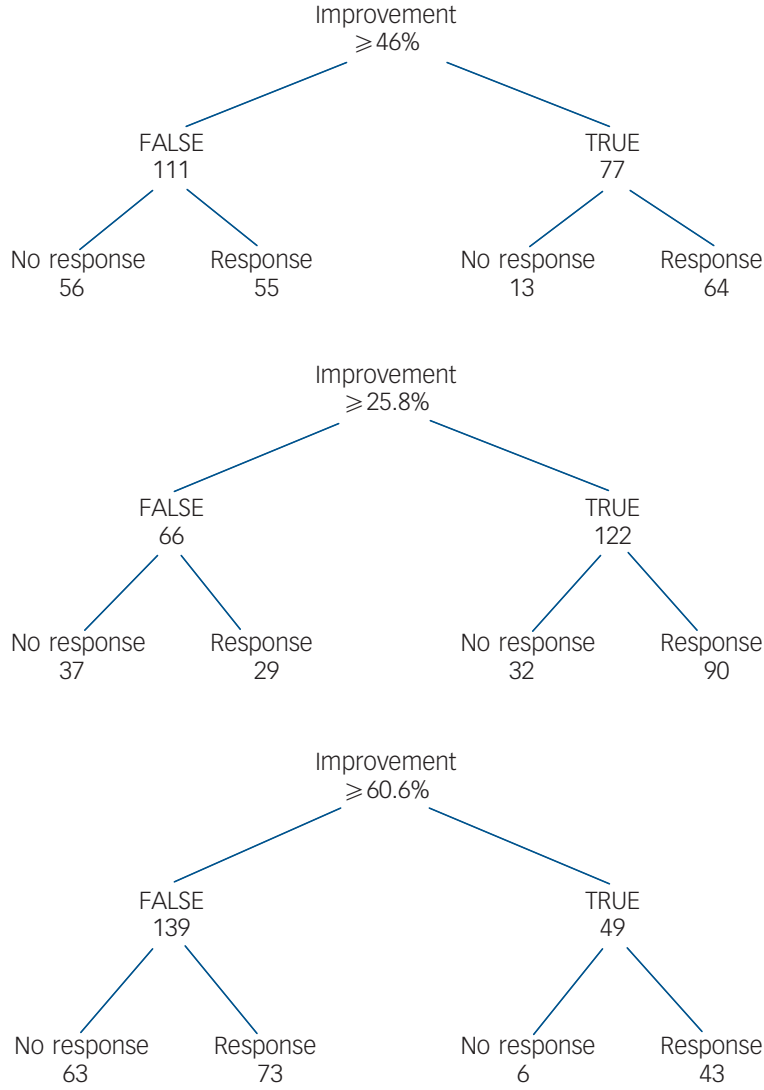

Fig. 5 Patients with early improvement and subsequent response/non-response.

The figure shows early improvers at week 2 who achieved the numerical cut-off, the lower cut-off of the proposed interval and upper cut-off of the proposed interval and those patients who concurrently achieved response/non-response.

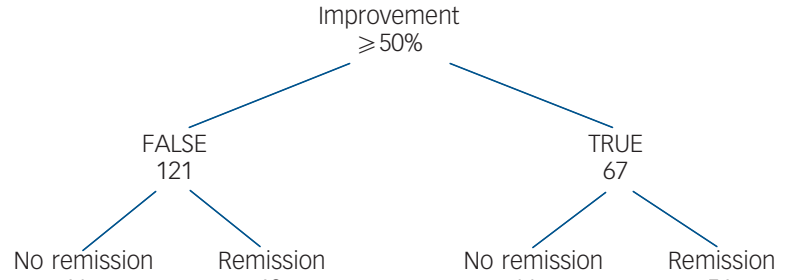

61
56
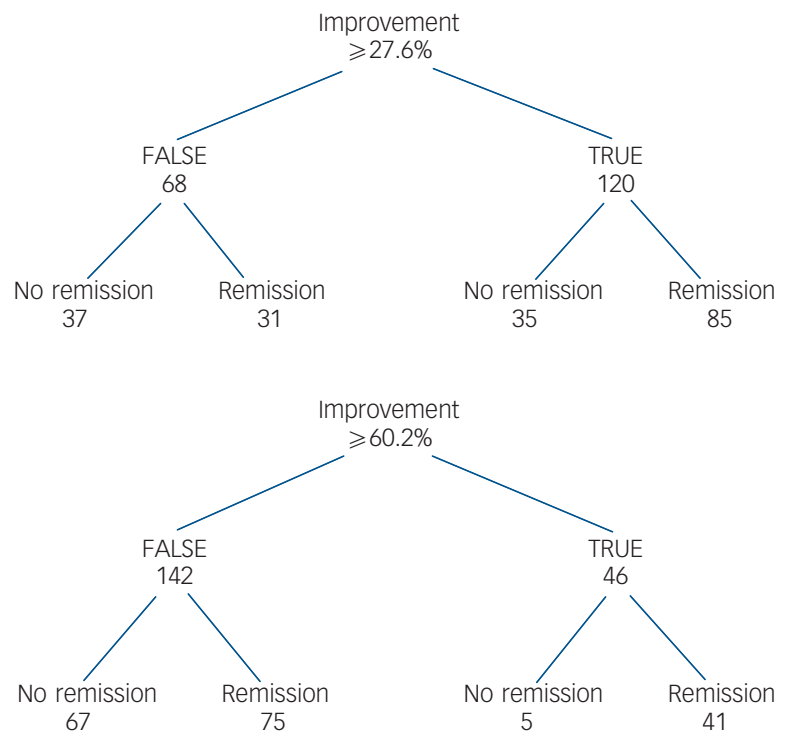

Fig. 6 Patients with early improvement and subsequent remission or non-remission.

This figure shows early improvers at week 2 who achieved the numerical cut-off, the This figure shows early improvers at week 2 who achieved the numerical cut-off, the
lower cut-off of the proposed interval and upper cut-off of the proposed interval and lower cut-off of the proposed interval and upper cut-off of the propose
those patients who concurrently achieved remission/non-remission.

which usually rely on a $20 \%$ improvement. ${ }^{17,32}$ In particular, the $60 \%$ improvement after two treatment weeks seems too strict at first glance, even for first-episode schizophrenia. However, in our study we found that $23 \%$ of the patients who had achieved a $60 \%$ PANSS total score improvement at week 2 were concurrently responders at week 8 , and that $22 \%$ of the patients were in remission (see Figs 5 and 6). This suggests that a 60\% improvement at week 2 predicting subsequent response and remission is not unrealistic in first-episode disorder, indicating that it might be more appropriate to choose stricter cut-offs in such cases, thereby maybe falsely 'overtreating' early responders rather than missing early non-responders.

The possibility of a comparison with other trials evaluating antipsychotic treatment response in a double-blind design with risperidone and haloperidol in first-episode schizophrenia is limited. Examining the time course of antipsychotic treatment response, Emsley et al found a total of $47 \%$ patients achieving a PANSS improvement of at least $20 \%$ by week $2 .^{33}$ In our trial $70 \%$ of the patients improved by at least $20 \%$ at week 2 . This discrepancy in response rates may be explained by several trialand protocol-specific reasons. ${ }^{33}$ Interestingly, when using sensitivity and specificity analysis in a naturalistic study design examining a heterogeneous patient population, Jäger et al found a 30\% PANSS total improvement at week 2 to best predict subsequent response. ${ }^{34}$ This also supports the conclusion that the proposed cut-off interval is a suitable landmark to evaluate early treatment improvement and thereby optimise treatment outcome.

\section{Clinical implications of using $30 \%$ improvement to predict outcome}

Our results imply that patients should improve by at least 30\% in PANSS total score to achieve subsequent response and remission. We did not find significant differences in the dosage applied comparing patients with or without $30 \%$ early improvement in the first two treatment weeks or in the type of antipsychotic drug. As can be seen in Fig. 2, clinicians intuitively treated those not showing early improvement with significantly higher doses from week 2 on. Interestingly, despite receiving significantly more antipsychotic medication, these patients still achieved response and remission significantly less often. This suggests that a dosage increase might not be enough, and that a change in antipsychotic compound or the implementation of further treatment strategies is necessary to ensure a satisfactory outcome. Future studies need to evaluate the proposed cut-off interval and to reassess the benefit of treatment adjustment for patients not improving initially.

\section{Strengths and limitations}

The strengths of our study include the fact that all patients examined had first-episode disorder, as well as the use of standardised assessment, the chance to test early improvement for both a typical and an atypical antipsychotic, and the doubleblind design. Limitations are the strict study protocol reducing the generalisability of the results, as well as the small number of participants. The time criterion of the applied consensus 
remission criteria was not considered. Unfortunately, it was only possible to evaluate early improvement and its predictive validity within the first 8 treatment weeks, thereby excluding long-term data. Although a simple dosage increase seemed an insufficient strategy for those who did not show early improvement, we did not examine whether a change of treatment in such patients would influence subsequent outcome, because the treatment applied at admission was continued. The flexible dosing again presents both an advantage, in meeting clinical practice, and a limitation, in eliminating examination of the time to respond to a specific dosage.

Rebecca Schennach-Wolff MD, Florian Hannes Seemüller MD, Andreas May, Department of Psychiatry and Psychotherapy, Ludwig Maximilians University, Munich Wolfgang Maier, MD, Professor of Psychiatry, Department of Psychiatry and Psychotherapy, University of Bonn; Stefan Klingberg, MD, Professor of Psychiatry, Department of Psychiatry and Psychotherapy, University of Tübingen; Isabella Heuser, MD, Professor of Psychiatry, Department of Psychiatry and Psychotherapy, Charité Berlin, Campus Benjamin Franklin, Berlin; Joachim Klosterkötter, MD, Professor of Psychiatry, Department of Psychiatry and Psychotherapy, University of Cologne; Markus Gastpar, MD, Professor of Psychiatry, Psychiatric Clinic, Fliedne Hospital, Berlin; Heinz Häfner, MD, Professor of Psychiatry, Central Institute of Mental Health, Mannheim; Heinrich Sauer, MD, Professor of Psychiatry, Department of Psychiatry and Psychotherapy, University of Jena; Frank Schneider, MD, Professor of Psychiatry, Department of Psychiatry and Psychotherapy, RWTH Aachen University: Wolfgang Gaebel, MD, Professor of Psychiatry, Department of Psychiatry and Psychotherapy, Heinrich Heine University, Düsseldorf; Markus Jäger, PD, HansJürgen Möller, MD, Professor of Psychiatry, Michael Riedel, PD, Department of Psychiatry and Psychotherapy, Ludwig Maximilians University, Munich, Germany

Correspondence: Dr Rebecca Schennach-Wolff, Department of Psychiatry and Psychotherapy, Ludwig-Maximilians-University, Nussbaumstrasse 7, Muenchen 80336, Germany. Email: rebecca.schennach-wolff@med.uni-muenchen.de

First received 9 Jun 2009, final revision 1 Dec 2009, accepted 8 Feb 2010

\section{Funding}

This work was supported by the German Federal Ministry for Education and Research BMBF (grants $01 \mathrm{Gl} 0032,01 \mathrm{Gl}$ 0232). Risperidone and haloperidol, and the blinding and randomisation procedure, were provided by Janssen-Cilag, Germany. This study was conducted within the framework of the German Research Network on Schizophrenia, which is funded by the German Federal Ministry for Education and Research.

\section{Acknowledgements}

The authors thank all those who were involved in the study, especially Mrs Thelma Coutts for editing the manuscript.

\section{References}

1 Agid O, Kapur S, Arenovich T, Zipursky RB. Delayed-onset hypothesis of antipsychotic action: a hypothesis tested and rejected. Arch Gen Psychiatry 2003; 60: 1228-35.

2 Correll CU, Malhotra AK, Kaushik S, McMeniman M, Kane J. Early prediction of antipsychotic response in schizophrenia. Am J Psychiatry 2003; 160 2063-5.

3 Leucht S, Shamsi SA, Busch R, Kissling W, Kane JM. Predicting antipsychotic drug response - replication and extension to six weeks in an international olanzapine study. Schizophr Res 2008; 101: 312-9.

4 Lin $\mathrm{CH}$, Chou LS, Lin CH, Hsu CY, Chen YS, Lane HY. Early prediction of clinical response in schizophrenia patients receiving the atypical antipsychotic zotepine. J Clin Psychiatry 2007; 68: 1522-7.

5 Kasper S. First-episode schizophrenia: the importance of early intervention and subjective tolerability. J Clin Psychiatry 1999; 60 (suppl 23): 5-9.

6 Emsley R, Oosthuizen PP, Kidd M, Koen L, Niehaus DJ, Turner HJ. Remission in first-episode psychosis: predictor variables and symptom improvement patterns. J Clin Psychiatry 2006; 67: 1707-12.

7 Emsley R, Rabinowitz J, Medori R. Remission in early psychosis: rates, predictors, and clinical and functional outcome correlates. Schizophr Res 2007; 89: 129-39.

8 Crespo-Facorro B, Pelayo-Teran JM, Perez-Iglesias R, Ramírez-Bonilla M, Martínez-García O, Pardo-García G, et al. Predictors of acute treatment response in patients with a first episode of non-affective psychosis: sociodemographics, premorbid and clinical variables. J Psychiatr Res 2007; 41: 659-66.
9 Schooler N, Rabinowitz J, Davidson M, Emsley R, Harvey PD, Kopala L, et al. Risperidone and haloperidol in first-episode psychosis: a long-term randomized trial. Am J Psychiatry 2005; 162: 947-53.

10 Gaebel W, Riesbeck M, Wolwer W, Klimke A, Eickhoff M, von Wilmsdorff M, et al. Maintenance treatment with risperidone or low-dose haloperidol in first-episode schizophrenia: 1-year results of a randomized controlled trial within the German Research Network on Schizophrenia. J Clin Psychiatry 2007; 68: 1763-74.

11 Möller HJ, Riedel M, Jäger M, Wickelmaier F, Maier W, Kühn KU, et al. Shortterm treatment with risperidone or haloperidol in first-episode schizophrenia: 8 -week results of a randomized controlled trial within the German Research Network on Schizophrenia. Int J Neuropsychopharmacol 2008; 11: 985-97.

12 American Psychiatric Association. Diagnostic and Statistical Manual of Mental Disorders (4th edn) (DSM-IV). APA, 1994.

13 Cording C. Conceptual aspects in development and implementation of basic psychiatric documentation [in German]. Psychiatr Prax 1998; 25: 175-8.

14 Kay SR, Opler LA, Lindenmayer JP. Reliability and validity of the positive and negative syndrome scale for schizophrenics. Psychiatry Res 1988; 23: 99-110.

15 Guy W. ECDEU Assessment Manual for Psychopharmacology. Revised DHEW Pub. (ADM). National Institute for Mental Health, 1976.

16 American Psychiatric Association. Diagnostic and Statistical Manual of Mental Disorders (3rd edn, revised) (DSM-III-R). APA, 1987.

17 Kinon BJ, Chen L, Scher-Svanum H, Stauffer VL, Kollack-Walker S, Sniadecki $\mathrm{JL}$, et al. Predicting response to atypical antipsychotics based on early response in the treatment of schizophrenia. Schizophr Res 2008; 102: $230-40$.

18 Obermeier M, Mayr A, Schennach-Wolff R, Seemüller F, Möller HJ, Riedel M. Should the PANSS be rescaled? Schizophr Bull 2009; [epub ahead of print]

19 Andreasen NC, Carpenter WT, Kane JM, Lasser RA, Marder SR, Weinberger DR, et al. Remission in schizophrenia: proposed criteria and rationale for consensus. Am J Psychiatry 2005; 162: 441-9.

20 Weinstein MC, Fineberg HV. Clinical Decisions Analysis. Saunders, 1980.

21 Fawcett T. An introduction to ROC analysis. Pattern Recognit Lett 2006; 27: 861-74.

22 R Development Core Team. A Language and Environment for Statistical Computing. Foundation for Statistical Computing, 2008 (http//: www.R-project.org)

23 Cumming G. Inference by eye: reading the overlap of independent confidence intervals. Stat Med 2009; 28: 205-20.

24 Mccreadie RG. Managing the first episode of schizophrenia: the role of new therapies. Eur Neuropsychopharmacol 1996; 6 (suppl 2): S3-5.

25 Chang YC, Lane $\mathrm{HY}$, Yang $\mathrm{KH}$, Huang $\mathrm{CL}$. Optimizing early prediction for antipsychotic response in schizophrenia. J Clin Psychopharmacol 2006; 26: 554-9.

26 Leucht S, Busch R, Kissling W, Kane M. Early prediction of antipsychotic nonresponse among patients with schizophrenia. J Clin Psychiatry 2007 68: $352-60$

27 Ceskova E, Prikryl R, Kasparek T, Ondrusova M. Psychopathology and treatment responsiveness of patients with first-episode schizophrenia. Neuropsychiatr Dis Treat 2005; 1: 179-85.

28 Boter H, Peuskens J, Libiger J, Fleischhacker WW, Davidson M, Galderisi S, et al. Effectiveness of antipsychotics in first-episode schizophrenia and schizophreniform disorder on response and remission: an open randomized clinical trial (EUFEST). Schizophr Res 2009; 115: 97-103.

29 Crespo-Facorro B, Perez-Iglesias R, Ramirez-Bonilla M, Martínez-García O, Llorca J, Luis Vázquez-Barquero J. A practical clinical trial comparing haloperidol, risperidone, and olanzapine for the acute treatment of firstepisode nonaffective psychosis. J Clin Psychiatry 2006; 67: 1511-21.

30 Emsley RA. Risperidone in the treatment of first-episode psychotic patients: a double-blind multicenter study. Risperidone Working Group. Schizophr Bull 1999; 25: 721-9.

31 Efron B, Tibshirani R. An Introduction to the Bootstrap. Monographs on Statistics and Applied Probability 57. Chapman \& Hall/CRC, 1994.

32 Scher-Svanum H, Nyhuis AW, Faries DE, Kinon BJ, Baker RW, Shekhar A. Clinical, functional, and economic ramifications of early nonresponse to antipsychotics in the naturalistic treatment of schizophrenia. Schizophr Bull 2008; 34: 1163-71.

33 Emsley R, Rabinowitz J, Medori R. Time course for antipsychotic treatment response in first-episode schizophrenia. Am J Psychiatry 2006; 163: 743-5.

34 Jäger $M$, Schmauß $M$, Laux $G$, Pfeiffer $H$, Naber $D$, Schmidt LG, et al. Early improvement as a predictor of remission and response in schizophrenia. Results from a naturalistic study. Eur Psychiatry 2009; 24: 501-6. 